\title{
Clinical spectrum, outcome and management of immune thrombocytopenia associated with myelodysplastic syndromes and chronic myelomonocytic leukemia
}

Haematologica 2021

Volume 106(5):1414-1422

\section{Correspondence:}

VINCENT JACHIET

vincent.jachiet@aphp.fr

Received: September 17, 2020.

Accepted: November 23, 2020.

Pre-published: February 25, 2021.

https://doi.org/10.3324/haematol.2020.272559

(C)2021 Ferrata Storti Foundation

Material published in Haematologica is covered by copyright. All rights are reserved to the Ferrata Storti Foundation. Use of published material is allowed under the following terms and conditions:

https://creativecommons.org//icenses/by-nc/4.0/legalcode. Copies of published material are allowed for personal or internal use. Sharing published material for non-commercial purposes is subject to the following conditions:

https://creativecommons.org/licenses/by-nc/4.0/legalcode, sect. 3. Reproducing and sharing published material for commercial purposes is not allowed without permission in writing from the publisher.
Vincent Jachiet, ${ }^{1}$ Guillaume Moulis, ${ }^{2}$ Jérome Hadjadj, ${ }^{3}$ Julie Seguier, ${ }^{4}$ Kamel Laribi, ${ }^{5}$ Nicolas Schleinitz, ${ }^{4}$ Norbert Vey, ${ }^{6}$ Karim Sacre, ${ }^{7}$ Bertrand Godeau, ${ }^{8}$ Odile Beyne-Rauzy, ${ }^{9}$ Romain Bouvet, ${ }^{10}$ Jonathan Broner, ${ }^{11}$ Natacha Brun, ${ }^{12}$ Thibault Comont, ${ }^{9}$ Clément Gaudin, ${ }^{13}$ Olivier Lambotte, ${ }^{14}$ Lenaïg Le Clech, ${ }^{15}$ Pierre Peterlin, ${ }^{16}$ Frédérique Roy-Peaud, ${ }^{17}$ Clémentine Salvado, ${ }^{18}$ Mathilde Versini, ${ }^{19}$ Françoise Isnard, ${ }^{20}$ Jean Emmanuel Kahn, ${ }^{21}$ Delphine Gobert, ${ }^{1}$ Lionel Adès, ${ }^{22}$ Pierre Fenaux, ${ }^{22}$ Olivier Fain ${ }^{1}$ and Arsène Mekinian ${ }^{1}$, on behalf of MINHEMON, GFM, CERECAI and CARMEN investigators group*

${ }^{1}$ Service de Médecine Interne and Inflammation-Immunopathology-Biotherapy Department (DMU 3iD), Hôpital Saint-Antoine, Assistance Publique - Hôpitaux de Paris, Sorbonne Université, Paris; ${ }^{2}$ Service de Médecine Interne, Centre Hospitalier Universitaire de Toulouse, Université de Toulouse, Toulouse; ${ }^{3}$ Department of Internal Medicine, Hôpital Cochin, Assistance Publique - Hôpitaux de Paris, Université de Paris, Paris; ${ }^{4}$ Département de Médecine Interne, Hôpital de la Timone, Assistance Publique Hôpitaux de Marseille, Aix Marseille Université, Marseille; ${ }^{5}$ Department of Hematology, Centre Hospitalier Le Mans, Le Mans; ${ }^{\circ}$ Hematology Department, Institut PaoliCalmettes, Aix-Marseille Université, Marseille; 'Departement de Médecine Interne, Hôpital Bichat, Assistance Publique - Hôpitaux de Paris, Université de Paris, Paris; ${ }^{8}$ Médecine Interne, Hôpital Henri Mondor, Assistance Publique - Hôpitaux de Paris, Université Paris-Est Créteil, Créteil; ${ }^{9}$ Department of Internal Medicine, Institut Universitaire du Cancer de Toulouse-Oncopole, Toulouse; ${ }^{10}$ Médecine Interne et Maladies Systémiques, Centre Hospitalier Universitaire Dijon Bourgogne, Dijon; ${ }^{11}$ Internal Médicine Department, Nîmes University Hospital, University of Montpellier, Nîmes; ${ }^{12}$ Service de Médecine Interne, Centre Hospitalier de Rodez, Rodez;

${ }^{13}$ Department of Oncogeriatric Medicine, University Hospital Purpan, Toulouse; ${ }^{14}$ Médecine Interne et Immunologie Clinique, Hôpital Bicêtre, Assistance Publique Hôpitaux de Paris, Université Paris Sud, Le Kremlin-Bicêtre; ${ }^{15}$ Department of Internal Medicine, Infectious Diseases and Hematology, Cornouaille Hospital Quimper, Quimper; ${ }^{16}$ Service d'Hématologie Clinique, Centre Hospitalier Universitaire de Nantes, Nantes; ${ }^{17}$ Service de Médecine Interne, Maladies Infectieuses et Tropicales, Centre Hospitalier Universitaire de Poitiers, Poitiers; ${ }^{18}$ Service de Maladies Infectieuses, Centre Hospitalier de Dax, Dax; ${ }^{19}$ Institut Arnault Tzanck, Saint Laurent du Var; ${ }^{20}$ Department of Clinical Hematology, Hôpital Saint-Antoine, Assistance Publique - Hôpitaux de Paris, Sorbonne Université, Paris; ${ }^{21}$ Service de Médecine Interne, Hôpital Ambroise Paré, Assistance Publique - Hôpitaux de Paris, Paris and ${ }^{22}$ Service d'Hématologie Clinique, Department of Hematology, Hôpital St. Louis, Assistance Publique - Hôpitaux de Paris, Université de Paris, Paris, France

* Listed in the Appendix

\section{ABSTRACT}

M yelodysplastic syndromes (MDS) and chronic myelomonocytic leukemia (CMML) are associated with systemic inflammatory or autoimmune diseases in $10-20 \%$ of cases. Immune thrombocytopenia (ITP) is among the reportedly associated diseases, but large studies assessing the association are lacking. It is unclear whether patients with MDS or CMML and ITP have a particular phenotype or require particular management. We, therefore, analyzed the clinical spectrum, outcome and therapeutic management of patients with ITP associated with MDS or CMML, in comparison to: (i) patients with primary ITP without MDS/CMML and (ii) patients with MDS/CMML without ITP. Forty-one patients with MDS/CMML-associated ITP were included, of whom 26 $(63 \%)$ had chronic ITP, 30 (73\%) had low-risk myelodysplasia and 24 $(59 \%)$ had CMML. An associated autoimmune disease was noted in ten $(24 \%)$ patients. In comparison to patients with primary ITP, patients with MDS/CMML-associated ITP had a higher rate of severe bleeding despite 
similar platelet counts at diagnosis. First-line treatment consisted of glucocorticoids (98\%) and intravenous immunoglobulins (56\%). Patients with primary ITP were more likely to respond to intravenous immunoglobulins than were patients with MDS/CMML-associated ITP. Response rates to second-line therapies were not statistically different between patients with primary ITP or MDS/CMML-associated ITP. Four $(10 \%)$ of the patients with MDS/CMML-associated ITP had multirefractory ITP whereas none of the primary ITP controls did so. After a median follow-up of 60 months, there was no difference in overall survival between patients with MDS/CMML-associated ITP or primary ITP. Leukemia-free-survival was significantly better in patients with MDS/CMML-associated ITP than in those with MDS/CMML without ITP. In conclusion, it appears that patients with MDS/CMML-associated ITP have a particular phenotype, with more severe bleeding than patients with primary ITP, a higher likelihood of multirefractory disease, but a similar response to primary ITP therapy except for intravenous immunoglobulins. Finally, compared to MDS/CMML patients without ITP, they are less likely to progress to having acute myeloid leukemia.

\section{Introduction}

Myelodysplastic syndromes (MDS) and chronic myelomonocytic leukemia (CMML) are clonal hematopoietic stem cell disorders characterized by ineffective and dysplastic hematopoiesis in the bone marrow leading to cytopenias and a risk of developing acute myeloid leukemia (AML). ${ }^{1}$ In 10 to $20 \%$ of cases, various systemic inflammatory or autoimmune diseases (SIAD) can be associated with MDS or CMML. ${ }^{2}$ The impact of these associated diseases on the survival and progression to acute leukemia of MDS/CMML patients remains controversial, but they can make therapy challenging. In addition to the most frequently reported SIAD (vasculitis, neutrophilic dermatoses, and polyarthritis), immune cytopenias have been documented in 1 to $16 \%$ of cases. ${ }^{3.5}$ Immune thrombocytopenia (ITP) is an immune-mediated acquired disorder defined by a transient or persistent decrease in the peripheral blood platelet count to below $100 \times 10^{9} / \mathrm{L}$ for which other causes of isolated thrombocytopenia have been excluded. ${ }^{6}$ ITP is characterized by autoimmune-mediated platelet destruction and impaired platelet production, which can lead to an increased risk of bleeding. Large studies analyzing the specific features, outcome and treatment of ITP in MDS/CMML are lacking. The aim of this study by the French Network of Dysimmune Disorders Associated with Hemopathies (MINHEMON) was to describe the clinical spectrum, therapeutic management and outcome of patients with ITP in the context of MDS/CMML in comparison to: (i) patients with primary ITP without MDS/CMML and (ii) patients with MDS/CMML without ITP.

\section{Methods}

\section{Patients}

We retrospectively collected data on patients with ITP associated with MDS/CMML diagnosed since January 1999 at 16 French departments of internal medicine and hematology. Physicians were asked by MINHEMON, the Reference Center for Autoimmune Cytopenias in Adults (CERECAI) and the French Society of Internal Medicine (SNFMI) to report cases of ITP associated with MDS or CMML. Some patients with ITP and CMML $(n=5)$ presented in this case series were described in a previous publication. ${ }^{7}$ Clinical, laboratory and immunological data at the time of diagnosis of MDS/CMML and ITP and during the follow-up were collected using a standardized form.
Patients were included if they fulfilled the following criteria: (i) age over 18 years; (ii) a diagnosis of ITP according to the international criteria; ${ }^{6}$ platelet count $<100 \times 10^{9} / \mathrm{L}$ on at least two separate occasions and the exclusion of other causes of thrombocytopenia; (iii) steroid-responsive thrombocytopenia (with response defined as any platelet count of at least $30 \times 10^{9} / \mathrm{L}$ and at least doubling of the baseline count, as described by Rodeghiero et al. ${ }^{\circ}$ ); (iv) a diagnosis of MDS or CMML, based on blood and bone marrow examinations, according to the 2016 World Health Organization classification; ${ }^{8}$ and (v) a maximum period of 10 years between the diagnoses of ITP and MDS/CMML.

The exclusion criteria were as follows: (i) patients with secondary ITP not linked to MDS (e.g., cases related to malignancy, chronic viral infection, primary immune deficiency or drugs); and (ii) lack of response to steroids.

Patients were classified using the Revised International Prognostic Scoring System (IPSS-R) and separated into two subgroups, low- and high-risk MDS, based on a cut-off IPSS-R score of 3.5 points. ${ }^{9}$ Secondary MDS refers to therapy-related MDS (occurring after chemotherapy or radiation therapy) or to MDS associated with another primary or acquired bone marrow disorder.

Bleeding was graded according to the bleeding score previously reported by Khellaf et al. ${ }^{10}$ Severe bleeding was defined as intracranial hemorrhage, overt gastrointestinal bleeding, severe menstrual bleeding, or macroscopic hematuria. ${ }^{11}$ Multirefractory ITP was defined as severe chronic ITP not responding to rituximab, splenectomy or a thrombopoietin receptor agonist (TPO-RA). ${ }^{12}$

This study was conducted in compliance with the Helsinki Declaration, upon a database of patients treated according to standard care and, therefore, no ethics approval was necessary according to French law (https://www.legifrance.gouv.fr/eli/decret/ 2017/5/9/AFSP1706303D/jo/texte). The database was declared with registration number $2218061 \mathrm{v} 0$ to the Commission Nationale de l'Informatique et des Libertés (CNIL).

\section{Criteria for immune thrombocytopenia response}

Assessment of the response to ITP treatments was time-specific to each treatment, as described by Rodeghiero et al. ${ }^{6}$ Complete response was defined as any platelet count of at least $100 \times 10^{9} / \mathrm{L}$. Partial response was defined as any platelet count between 30 and $100 \times 10^{9} / \mathrm{L}$ and at least doubling of the baseline count. No response was defined as any platelet count lower than $30 \times 10^{9} / \mathrm{L}$ or less than doubling of the baseline count. The definitions of partial response and complete response required concurrent resolution of bleeding symptoms. 
Comparison with two prospective cohorts of patients with only immune thrombocytopenia or with only myelodysplastic syndrome/chronic myelomonocytic

\section{leukemia}

Patients with MDS/CMML-associated ITP were matched for age ( \pm 5 years) and sex to patients with primary ITP included between 2013 and 2019 in the multicenter Cytopénies Autoimmunes: Registre Midi-PyrénéEN (CARMEN) registry at a 1:2 ratio. CARMEN is a registry, established in June 2013, that follows prospectively incident ITP adult patients ( $\geq 18$ years) in the MidiPyrénées region (southern France, 3 million inhabitants). ${ }^{13,14}$ The absence of myelodysplasia in all primary ITP controls in this study was confirmed by bone marrow examinations.

Patients with MDS/CMML-associated ITP were also matched for age ( \pm 5 years), sex, type of disorder (MDS or CMML) and IPSS$\mathrm{R}$ score $\left(\leq\right.$ or $\left.>3.5^{9}\right)$ to patients with MDS/CMML without ITP beginning in 2003 in the multicenter Groupe Francophone des Myélodysplasies (GFM) registry of MDS/CMML at a 1:4 ratio.

\section{Statistical analysis}

Continuous variables are presented as the mean \pm standard deviation or as the median (range), as appropriate. Qualitative variables are presented as a number (\%). The Mann-Whitney test and $t$-test were used to compare continuous variables, and the $\chi^{2}$ and Fisher exact tests were used to compare qualitative variables. Overall survival was calculated from the date of MDS/CMML diagnosis to death or the last date of follow-up. Leukemia-free survival was calculated from the date of MDS/CMML diagnosis to the date of transformation to AML. Overall and leukemia-free survival were analyzed with the log-rank test, and the results are expressed using Kaplan-Meier methods. A $P$-value $<0.05$ was considered statistically significant. Statistical analyses were performed using GraphPad Prism 5.0 for Mac (GraphPad Software ${ }^{\mathrm{TM}}$, La Jolla, CA, USA).

\section{Results}

\section{Characteristics of patients with myelodysplastic syndrome/chronic myelomonocytic \\ leukemia-associated immune thrombocytopenia}

Between January 1999 and July 2019, we screened 77 patients with MDS/CMML and ITP and ultimately included 41 patients from 16 French hospitals (Figure 1). The median age at diagnosis was 77 years (range, 35-92) in the MDS/CMML group with ITP, and $41 \%$ of the patients were women (Table 1). The median IPSS-R score was 3 (range, 1-5), and 30 (73\%) patients had low-risk MDS. ITP was diagnosed concomitantly with MDS/CMML $( \pm 3$ months) in $17(41 \%)$ patients. The diagnosis of ITP preceded that of MDS/CMML (-116 to - 6 months) in 16 $(39 \%)$ patients and occurred after the diagnosis of

Table 1. Baseline characteristics and outcome of patients with myelodysplastic syndrome/chronic myelomonocytic leukemia (MDS/CMML)-associated immune thrombocytopenia (ITP), MDS/CMML without ITP and primary ITP.

\begin{tabular}{|c|c|c|c|c|}
\hline & $\begin{array}{l}\text { MIDS/CMMLIIIP } \\
n=41\end{array}$ & $\begin{array}{c}\text { Primaly IIIP } \\
\qquad=7.5 \\
\text { N }(\%) \text { or meditan [range] }\end{array}$ & $\begin{array}{l}\text { MDS/CMML without IIP } \\
\qquad=200\end{array}$ & $P$ \\
\hline Age at diagnosis, years & $77[35-92]$ & $76[35-92]$ & 78 [28-92] & 0.33 \\
\hline Female sex & $17(41)$ & $29(39)$ & $75(38)$ & 0.89 \\
\hline $\begin{array}{l}\text { ITP features } \\
\text { Platelet count at ITP diagnosis, x } 10^{9} / \mathrm{L} \\
\text { Khellaf bleeding score }>8 \\
\text { Severe bleeding } \\
\text { Antiplatelet antibodies } \\
\text { Direct antiglobulin test } \\
\text { Antinuclear antibodies } \\
\text { Polyclonal hypergammaglobulinemia }\end{array}$ & $\begin{array}{c}15[1-90] \\
11 / 38(29) \\
8 / 31(26) \\
7 / 16(44) \\
12 / 22(55) \\
12 / 29(41) \\
12 / 34(35)\end{array}$ & $\begin{array}{c}11[1-91] \\
21 / 75(28) \\
3 / 75(4) \\
- \\
- \\
26 / 57(46) \\
9 / 51(18)\end{array}$ & $\begin{array}{c}137 \text { [8-1488] } \\
- \\
- \\
- \\
- \\
- \\
-\end{array}$ & $\begin{array}{c}<0.01^{*} \\
0.92 \\
0.01^{*} \\
- \\
- \\
0.71 \\
0.07\end{array}$ \\
\hline $\begin{array}{l}\text { MDS/CMML features at inclusion } \\
\text { MDS } \\
\text { CMML } \\
\text { Platelet count, } x 10^{\circ} / \mathrm{L} \\
\text { Hemoglobin, } \mathrm{g} / \mathrm{dL} \\
\text { Neutrophils, } \mathrm{x} 10^{9} / \mathrm{L} \\
\text { Bone marrow blasts, } \% \\
\text { Abnormal karyotype } \\
\text { Low-risk MDS/CMML } \\
\text { IPSS } \\
\text { IPSS-R } \\
\text { Secondary MDS } \\
\text { MDS treatment (except EPO and transfusion) } \\
\text { Number of treatment lines for MDS }\end{array}$ & $\begin{array}{c}17(41) \\
24(59) \\
15[1-90] \\
11.8[5-15] \\
3.9[1.2-23] \\
3[0-13] \\
10 / 34(29) \\
30 / 41(73) \\
0.5[0-2] \\
3[1-5] \\
4 / 40(10) \\
9 / 41(22) \\
1[1-2]\end{array}$ & $\begin{array}{c}11 \text { [1-91] } \\
- \\
- \\
- \\
- \\
- \\
- \\
- \\
- \\
- \\
-\end{array}$ & $\begin{array}{c}84(42) \\
116(58) \\
137[8-1488] \\
10.9[6-16] \\
3.9[0.3-68] \\
3[0-29] \\
63 / 197(32) \\
155 / 200(78) \\
0.5[0-3.5] \\
2.5[0-8] \\
14 / 97(14) \\
45 / 200(23) \\
1[1-3]\end{array}$ & $\begin{array}{c}0.95 \\
<0.01^{*} \\
0.14 \\
0.72 \\
0.26 \\
0.73 \\
0.55 \\
0.66 \\
0.39 \\
0.14 \\
0.94 \\
0.91\end{array}$ \\
\hline $\begin{array}{l}\text { Outcome } \\
\text { Follow-up, months } \\
\text { AML transformation } \\
\text { Deaths } \\
\text { Deaths related to MDS/CMML or a specific therapy }\end{array}$ & $\begin{array}{l}66[1-176] \\
4 / 41(10) \\
17 / 41(41) \\
13 / 17(76) \\
\end{array}$ & $\begin{array}{l}- \\
- \\
- \\
-\end{array}$ & $\begin{array}{c}23[0-106] \\
15 / 200(8) \\
43 / 200(22) \\
18 / 43(42)\end{array}$ & $\begin{array}{c}<0.01^{\dagger} \\
0.63 \\
0.01^{\dagger} \\
0.02^{\dagger}\end{array}$ \\
\hline
\end{tabular}

ITP: immune thrombocytopenia; MDS: myelodysplastic syndrome; CMML: chronic myelomonocytic leukemia; IPSS: International Prognostic Scoring System; IPSS-R: Revised International Prognostic Scoring System; EPO: erythropoietin;AML: acute myeloid leukemia. ${ }^{*} P<0.05$ : MDS/CMML-associated ITP versus primary ITP; ${ }^{\dagger} P<0.05$ : MDS/CMML-associated ITP versus MDS/CMML without ITP; ${ }^{\ddagger} P<0.05$ : MDS/CMML-associated ITP versus primary ITP versus MDS/CMML without ITP. 
MDS/CMML (+4 to +111 months) in eight (20\%) patients (Online Supplementary Figure S1). The median platelet count was $15 \times 10^{9} / \mathrm{L}$ (range, 1-90) at ITP diagnosis, and the nadir platelet count was $8 \times 10^{9} / \mathrm{L}$. Twenty-nine percent $(11 / 38)$ of the patients had a high bleeding score (Khellaf score $>8$ ). Platelet transfusion therapy was ineffective in $78 \%(14 / 18)$ of the patients. An isotopic measurement of platelet lifespan carried out in four patients revealed a major reduction in the average platelet lifespan for all patients, with a median of 2.75 (range, 1-4) days. The assessment of the site of platelet destruction revealed a purely or predominantly splenic pattern for two patients and a mixed (hepatic and splenic) pattern for the other two patients. Antiplatelet antibodies detected by the monoclonal antibody immobilization of platelet antigens (MAIPA) assay were found in seven of $16(44 \%)$ patients (4 patients with a positive direct MAIPA only, and 3 patients with both direct and indirect positive MAIPA), and autoimmune hemolytic anemia was observed in five patients.

\section{Comparison of patients with immune thrombocytopenia with or without myelodysplastic syndrome/chronic myelomonocytic leukemia}

MDS/CMML-associated ITP patients were matched to 75 primary ITP controls without MDS/CMML (median age: 76 years [range, 35-92]; 39\% women) from the CARMEN registry (Table 1$)$. The platelet count at the time of ITP diagnosis was not significantly different between patients in the MDS/CMML-associated ITP group (15 $\mathrm{x}$ $10^{\circ} / \mathrm{L}$ [range, 1-90]) and those in the primary ITP group (11 $\times 10^{\circ} / \mathrm{L}$ [range, 1-91]; $P=0.39$ ), with similar rates of a high hemorrhagic score (Khellaf score >8) (29\% vs. 28\%, $P=0.92)$. However, MDS/CMML-associated ITP patients had a higher incidence of severe bleeding ( $26 \%$ vs. $4 \%$, $P=0.0009)$ involving the central nervous system $(n=3)$, gastrointestinal tract $(n=2)$ and other sites $(n=3)$. Polyclonal hypergammaglobulinemia ( $\gamma$-globulin level $>14 \mathrm{~g} / \mathrm{L}$ ) tended to be more frequent in the MDS/CMML-associated ITP group $(35 \%)$ than in the primary ITP group $(18 \%)(P=0.07)$.
Comparison of patients with myelodysplastic syndrome/chronic myelomonocytic leukemia with and without immune thrombocytopenia

MDS/CMML-associated ITP patients were matched to $200 \mathrm{MDS} / \mathrm{CMML}$ controls without ITP (median age: 78 years [range, 28-92]; 38\% women) from the GFM registry (Table 1). The distribution of MDS and CMML subtypes was similar in the two groups (MDS/CMML with or without ITP) (Online Supplementary Figure S2). The most frequent $\mathrm{MDS}$ subtypes were $\mathrm{MDS}$ with multilineage dysplasia $53 \%$ of cases of MDS/CMML with associated ITP and $39 \%$ of cases of MDS/CMML without ITP), MDS with single lineage dysplasia $(29 \%$ and $17 \%$, respectively) and MDS with excess blasts (12\% and $15 \%$, respectively). The proportion of secondary MDS was not different between groups, accounting for $10 \%$ of cases of MDS/CMML with ITP (chemotherapy [ $n=3]$, exposure to solvents $[n=1]$ or radioactivity $[n=1]$ ) and $14 \%$ of those with MDS/CMML without ITP.

MDS/CMML patients with ITP had a lower platelet count than MDS/CMML patients without ITP (median $15 \times 10^{9} / \mathrm{L}$ vs. $137 \times 10^{9} / \mathrm{L}, P<0.0001$ ), without significant differences concerning hemoglobin levels, neutrophil and monocyte counts, the number of bone marrow blasts and the median IPSS-R score. The rates of karyotypic abnormality were similar between groups (29\% vs. $32 \%$, $P=0.73)$. The most frequent abnormalities were $20 q(n=4)$ and $Y(n=4)$ deletions. The frequency of $20 q$ deletion in the group with MDS/CMML and ITP was higher than that in the group with MDS/CMML without ITP $(40 \%$ vs. $6 \%$, respectively, $P=0.002$ ). Approximately $20 \%$ of patients in each group $22 \%$ of the group with MDS/CMML and ITP; $23 \%$ of the group with MDS/CMML without ITP) received a specific hematologic treatment for myelodysplasia: hydroxyurea $(n=5 / 41$ in the MDS/CMML with ITP group and $n=25 / 200$ in the MDS/CMML without ITP group), azacytidine ( $n=4 / 41$ and none, respectively), allogeneic bone marrow transplant ( $n=1 / 41$ and $n=4 / 200$, respectively) or other therapies ( $n=2 / 41$ and $n=11 / 200$, respectively).

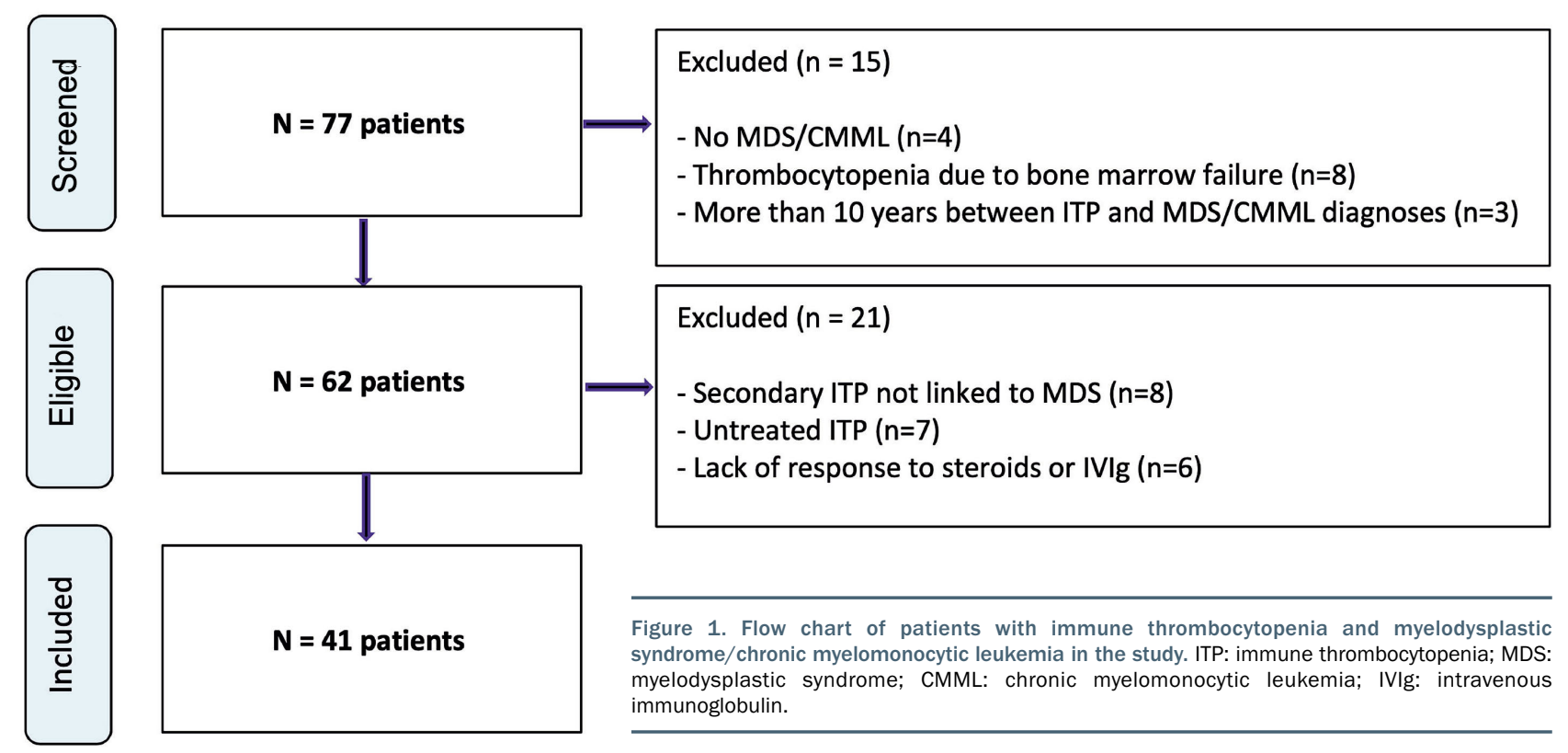


Management of thrombocytopenia in patients with myelodysplastic syndrome/chronic myelomonocytic leukemia-associated immune thrombocytopenia

All patients received a specific treatment for ITP, either for a platelet count $<30 \times 10^{9} / \mathrm{L}$ without a hemorrhagic syndrome $(44 \%)$, a hemorrhagic syndrome $(41 \%)$, concomitant antithrombotic medication or invasive procedure $(10 \%)$ and associated autoimmune hemolytic anemia $(5 \%)$. The response rates for each therapeutic strategy are indicated in Table 2. First-line treatment consisted of glucocorticoids and intravenous immunoglobulins (IVIg) $(56 \%)$, with higher response rates to IVIg in patients with primary ITP than in those with MDS/CMML-associated ITP $(90 \% v$ vs. $61 \%$, respectively, $P=0.0003$ ). ITP relapse rates after firstline therapy were significantly higher in the group with MDS/CMML-associated ITP (69\% after glucocorticoids and $77 \%$ after IVIg) than in the group with primary ITP ( $42 \%$ after glucocorticoids and $43 \%$ after $\operatorname{IVIg})(P=0.009)$. Second-line treatment consisted of a TPO-RA (68\%), danazol (44\%), rituximab (40\%), dapsone (20\%) or splenectomy $(13 \%)$ (Online Supplementary Figure S3). The mean number of immunosuppressive treatments used in second-line therapy was similar in the two groups $(2.1 \pm 1.5$ in the group with MDS/CMML-associated ITP vs. $1.6 \pm 0.8$ in the group with primary ITP; $P=0.42$ ), and approximately $40 \%$ of patients in each group received at least two different second-line treatments. The efficacy of each second-line treatment was comparable between the two groups, as illustrated in Figure 2. In the MDS/CMML-associated ITP group, the median duration of exposure was 18 months (range, 629) after rituximab, 14 months (range, 1-46) with TPO-RA, 6 months (range, 4-28) with danazol and 6 months (range, 5-6) with dapsone, with no significant differences between the durations of exposure to the different therapies.

Among the three patients with concomitant ITP and MDS/CMML treated with azacytidine (for MDS/CMML indications and two for refractory ITP), a complete hematologic response concomitant to the ITP complete response was noted in one patient. ${ }^{15}$

Unlike patients with primary ITP, four $(10 \%)$ patients with MDS/CMML-associated ITP presented with multirefractory ITP without simultaneous progression of the underlying myelodysplasia (confirmed by bone marrow aspiration and karyotype analysis in all four refractory cases).

\section{Outcome}

The median follow-up was 66 months (range, 1-176) in the group with MDS/CMML-associated ITP, 23 months (range, 0-106) in the group with MDS/CMML without ITP and 10 months (range, 0-53) in the group with primary ITP. There was no difference in the overall survival of patients with MDS/CMML-associated ITP and those with primary ITP (log-rank test $P=0.15$, median overall survival not reached in any group) (Figure 3A). The leukemia-free survival of patients with MDS/CMML-associated ITP was better than that of patients with MDS/CMML without ITP (log-rank test $P=0.05$, median leukemia-free survival not reached in any group) (Figure $3 \mathrm{~B}$ ). The four ITP patients who developed AML had CMML.

\section{Discussion}

From this multicenter study, we made the following observations: (i) patients with MDS/CMML-associated
ITP had a higher incidence of severe bleeding, despite similar platelet levels, than patients with primary ITP, which is probably explained by associated dysmegakaryopoiesis and platelet dysfunction in the former group; (ii) ITP was observed mostly in patients with low-risk MDS, according to the IPSS-R classification; (iii) the presence of a $20 \mathrm{q}$ deletion was more frequent among MDS/CMML patients with ITP than among those without ITP; (iv) compared to patients with primary ITP, patients with MDS/CMMLassociated ITP were characterized by a lower response rate to IVIg, more frequent relapses after first-line therapy and a multirefractory profile; and (v) compared to MDS/CMML patients without ITP, patients with MDS/CMML-associated ITP did not have a worse overall survival and the risk of AML progression was low.

Up to $20 \%$ of patients with MDS or CMML experience a SAID. ${ }^{3}$ These manifestations are part of a large and heterogeneous group of disorders. Among the various autoimmune or inflammatory disorders related to MDS/CMML, ITP is rare. Among the $61 \mathrm{MDS} / \mathrm{CMML}$

Table 2. Treatments for immune thrombocytopenia (ITP) and response rates in patients with myelodysplastic syndrome/chronic myelomonocytic leukemia-associated ITP and in those with primary ITP.

\begin{tabular}{|c|c|c|c|}
\hline & $\begin{array}{c}\text { MDS/CMML- } \\
\text { associated IIP } \\
n=41\end{array}$ & $\begin{array}{c}\text { Primainy } \\
\begin{array}{c}n=7 \\
n=75\end{array}\end{array}$ & P-value \\
\hline First-line treatment, n (\%) & $41 / 41(100)$ & $73 / 75(97)$ & 0.30 \\
\hline Glucocorticoids & $40 / 41(98)$ & $72 / 73(99)$ & 0.69 \\
\hline CR & $18 / 40(45)$ & $39 / 67(58)$ & 0.19 \\
\hline PR & $19 / 40(48)$ & $25 / 67(37)$ & 0.30 \\
\hline NR & $3 / 40(8)$ & $3 / 67(5)$ & 0.52 \\
\hline IVIg & $23 / 41(56)$ & $43 / 73(59)$ & 0.77 \\
\hline CR & $7 / 23(30)$ & $21 / 39(54)$ & 0.08 \\
\hline PR & $7 / 23(30)$ & $14 / 39(36)$ & 0.67 \\
\hline NR & $9 / 23(39)$ & $4 / 39(10)$ & 0.01 \\
\hline Second-line treatment, $\mathrm{n}(\%)$ & 25/41 (61) & $32 / 75(43)$ & 0.06 \\
\hline TPO-RA & $17 / 25(68)$ & $16 / 32(50)$ & 0.18 \\
\hline CR & $13 / 17(76)$ & $11 / 15(73)$ & 0.86 \\
\hline PR & $3 / 17(18)$ & $4 / 15(27)$ & 0.56 \\
\hline NR & $1 / 17(6)$ & 0 & 0.38 \\
\hline Danazol & $11 / 25(44)$ & $4 / 32(13)$ & 0.01 \\
\hline $\mathrm{CR}$ & $5 / 11(45)$ & $3 / 4(75)$ & 0.37 \\
\hline PR & $1 / 11(9)$ & 0 & 0.65 \\
\hline NR & $5 / 11(45)$ & $1 / 4(25)$ & 0.54 \\
\hline Rituximab & $10 / 25(40)$ & $15 / 32(47)$ & 0.61 \\
\hline CR & $3 / 9(33)$ & $6 / 9(67)$ & 0.19 \\
\hline PR & $2 / 9(22)$ & 0 & 0.58 \\
\hline NR & $4 / 9(44)$ & $3 / 9(33)$ & 0.14 \\
\hline Dapsone & $5 / 25(20)$ & $7 / 32(22)$ & 0.87 \\
\hline CR & $2 / 4(50)$ & $4 / 7(57)$ & 0.9 \\
\hline PR & $2 / 4(50)$ & $2 / 7(29)$ & 0.5 \\
\hline NR & 0 & $1 / 7(14)$ & 0.48 \\
\hline Splenectomy & $4 / 25(16)$ & 0 & 0.02 \\
\hline CR & $1 / 4(25)$ & - & - \\
\hline PR & $1 / 4(25)$ & - & - \\
\hline NR & $2 / 4(50)$ & - & - \\
\hline Other treatment & $5 / 25(20)$ & $10 / 32(31)$ & 0.35 \\
\hline $\begin{array}{l}\text { Number of second-line } \\
\text { treatments for ITP, mean (SD) }\end{array}$ & $2.1(1.5)$ & $1.6(0.8)$ & 0.42 \\
\hline Multirefractory ITP n(\%) & $4 / 41(10)$ & 0 & 0.01 \\
\hline
\end{tabular}

ITP: immune thrombocytopenia; MDS: myelodysplastic syndrome; CMML: chronic myelomonocytic leukemia; CR: complete response; PR: partial response; NR: no response; IVIg: intravenous immunoglobulin; TPO-RA: thrombopoietin receptor agonist; Other treatment: among vinca alkaloids, hydroxychloroquine, cyclosporine A, mycophenolate mofetil and azathioprine; SD: standard deviation. 
patients with a platelet count $<70 \times 10^{\circ} / \mathrm{L}$ in this study, $15 \%(n=9)$ had a reduced platelet lifespan, and six were treated with splenectomy, allowing a platelet response in three patients. ${ }^{16}$ In another series, 3.3\% $(n=46)$ of 1,408 MDS patients developed ITP. ${ }^{4}$ Among 2,882 French ITP patients, $2.3 \% \quad(n=67)$ had MDS, suggesting a role for MDS in the peak incidence of ITP in the elderly. ${ }^{17}$ In another French series of 565 ITP patients, $1.4 \% \quad(n=8)$ had CMML.? Among 62 MDS patients, Braun et al. reported that an isolated $20 \mathrm{q}$ deletion, which is associated with a good prognosis, was associated with a lower platelet count than that among MDS patients without a $20 \mathrm{q}$ deletion. $^{18}$
The main difficulty in the setting of MDS/CMML is to distinguish immune-related peripheral thrombocytopenia from that of central origin (i.e., due to bone marrow failure). Arguments for the peripheral immune origin of thrombocytopenia in MDS could be supported by the existence of common immune-mediated pathophysiological mechanisms, in particular humoral and cellular immune activation directed against both peripheral blood cells and bone marrow precursors, or impaired megakaryopoiesis attributed to autoantibodies cross-reacting against platelet glycoproteins or cytokine dysregulation. ${ }^{19}$

${ }^{22}$ Barcellini et al. hypothesized a shift from autoimmunity against circulating blood cells to bone marrow precursors,

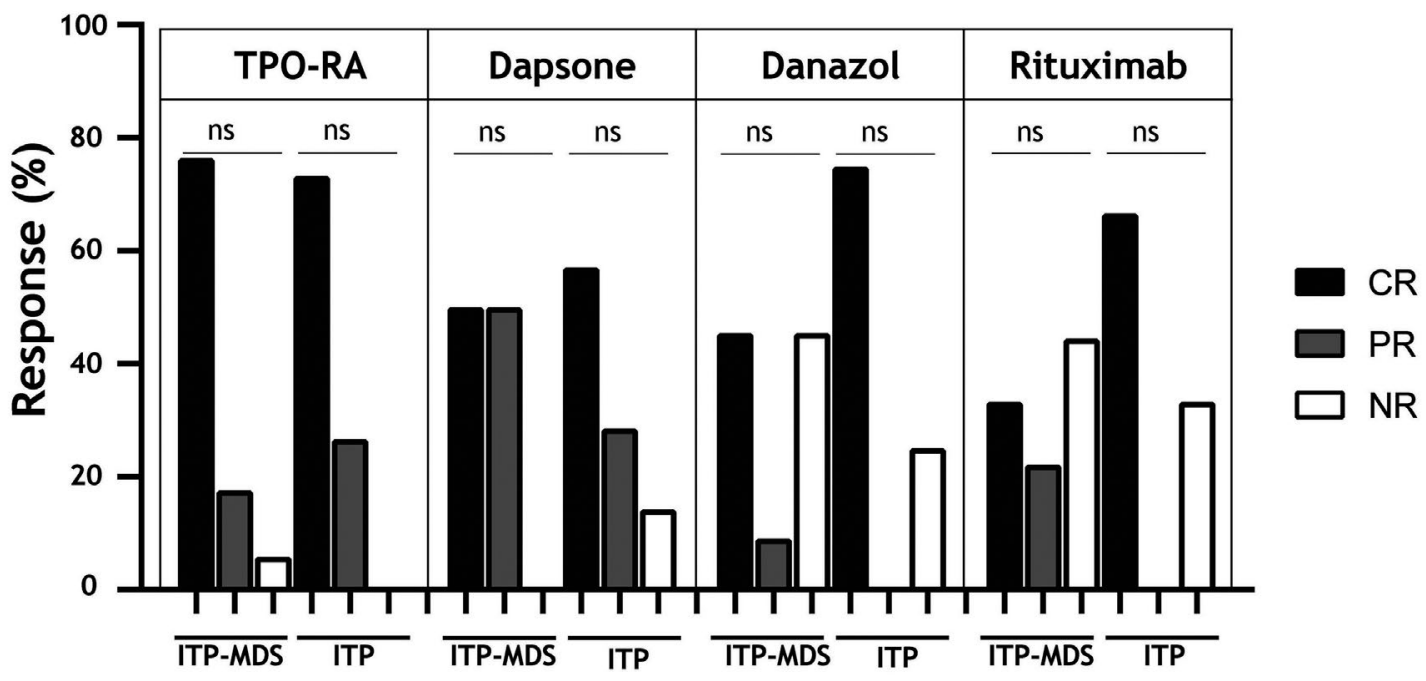

Figure 2. Response rates to second-line treatment in patients with myelodysplastic syndrome/chronic myelomonocytic leukemia and primary immune thrombocytopenia. ITP: immune thrombocytopenia; MDS: myelodysplastic syndrome; CMML: chronic myelomonocytic leukemia; TPO-RA: thrombopoietin receptor agonist; CR: complete response; PR: partial response; NR: no response; ITP: immune thrombocytopenia; MDS: myelodysplastic syndrome.

A

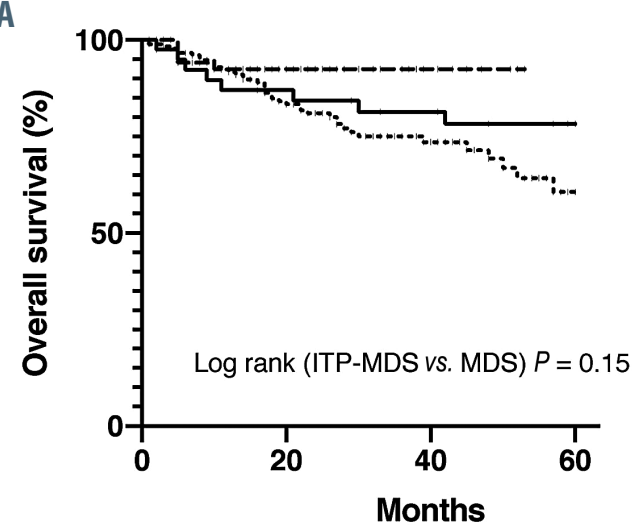

N. of subjects at risk

\begin{tabular}{lcccc}
\hline ITP-MDS & 41 & 33 & 28 & 22 \\
\hline MDS & 185 & 112 & 44 & 12 \\
\hline ITP & 88 & 35 & 10 & 0 \\
\hline
\end{tabular}

B

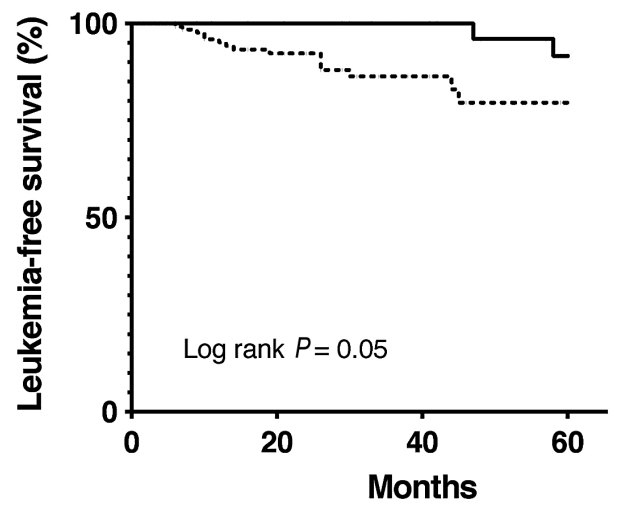

N. of subjects at risk

\begin{tabular}{lcccc}
\hline ITP-MDS & 41 & 33 & 28 & 20 \\
\hline MDS & 141 & 84 & 29 & 7 \\
\hline
\end{tabular}

Figure 3. Overall survival (A) and leukemia-free survival (B) of patients with myelodysplastic syndrome/chronic myelomonocytic leukemia (MDS/CMML)-associated immune thrombocytopenia (ITP), MDS/CMML without ITP and primary ITP. ITP: immune thrombocytopenia; MDS: myelodysplastic syndrome; CMML: chronic myelomonocytic leukemia. 
leading to an insufficient marrow compensatory response, a progressive/variable degree of bone marrow dysplasia, and ultimately overt bone marrow failure. ${ }^{19}$ Even if none of these features is pathognomonic of ITP, the probable peripheral origin of thrombocytopenia in MDS/CMML was based, in our study, on the following: (i) a clear-cut response, either partial or complete, to glucocorticoids; (ii) a discrepancy between an increased megakaryocyte count and profound thrombocytopenia; (iii) prominent dysimmune features in most cases, including positive antiplatelet antibodies, polyclonal hypergammaglobulinemia and other dysimmune diseases and/or autoantibodies; (iv) the lack of efficacy of platelet transfusions; and (v) mainly lowrisk underlying MDS. Isotopic measurement of platelet lifespan and assessment of the site of platelet destruction might help to predict the efficacy of splenectomy in ITP patients ${ }^{24-26}$ but such investigations are not usually performed routinely in France. Mahevas et al. recently showed that platelet kinetic studies could be used to predict the efficacy of splenectomy even in ITP patients treated with TPO-RA. ${ }^{27}$ Furthermore, Bourgeois et al. showed that $15 \%$ of low-risk MDS patients had peripheral platelet destruction, as demonstrated by isotopic autologous platelet kinetic studies. ${ }^{16}$ However, the small proportion of patients who have benefited from platelet scintigraphy does not allow the results to be generalized in this population of patients with MDS/CMML-associated ITP.

Finally, the most striking conclusion from our study is that ITP in the setting of MDS/CMML could be raised as a hypothesis to explain the thrombocytopenia in some cases and the benefit of immunomodulation could be tested in patients who do not need a specific treatment for their MDS.

The most challenging issue is the management of pre- sumed immune peripheral cytopenias in the setting of MDS/CMML (Figure 4). Few studies have assessed the outcome and management of MDS/CMML-associated ITP. The previous French case series of eight patients showed better response rates to IVIg among patients with primary ITP than among patients with MDS/CMMLassociated ITP. ${ }^{15}$ In this population of elderly patients, TPO-RA seem to be an interesting therapy because of their efficacy in our series and an acceptable safety profile. In a meta-analysis, Dodillet et al. found that treatment with TPO-RA resulted in a lower number of MDS patients suffering from bleeding events. Although some studies have highlighted the potential risk of TPO-RA in accelerating leukemic progression or an increase of blasts in MDS patients, ${ }^{28-30}$ more recent data from randomized controlled trials $^{31-33}$ and meta-analyses ${ }^{34,35}$ did not seem to confirm this. Moreover, preclinical studies found that eltrombopag could inhibit leukemic cell growth in tissue culture and in animal models of leukemia. ${ }^{23,36-39}$ While waiting for further clinical studies, close monitoring of peripheral blood counts and bone marrow and cytogenetic evaluations should be performed in patients who are on TPO-RA. Beyond their effect of stimulating megakaryopoiesis, additional mechanisms of action of TPO-RA include immunomodulating activity, such as the modulation of $\mathrm{T}$ regulatory cell ${ }^{21}$ and restoration of the $\mathrm{Fc}-\gamma$ receptor balance in phagocytes..$^{40}$ Finally, the efficacy of TPO-RA in patients with MDS/CMML-associated ITP is probably due to the fact that these drugs are efficient in both ITP and MDS. The efficacy of azacytidine for immune ITP must be interpreted with caution because of the small number of patients treated. An ongoing phase II French trial is currently assessing the efficacy and safety of azacytidine in various steroid-dependent/refractory

\section{Thrombocytopenia $<30 \times 10 \% / L$ during MDS/CMML}

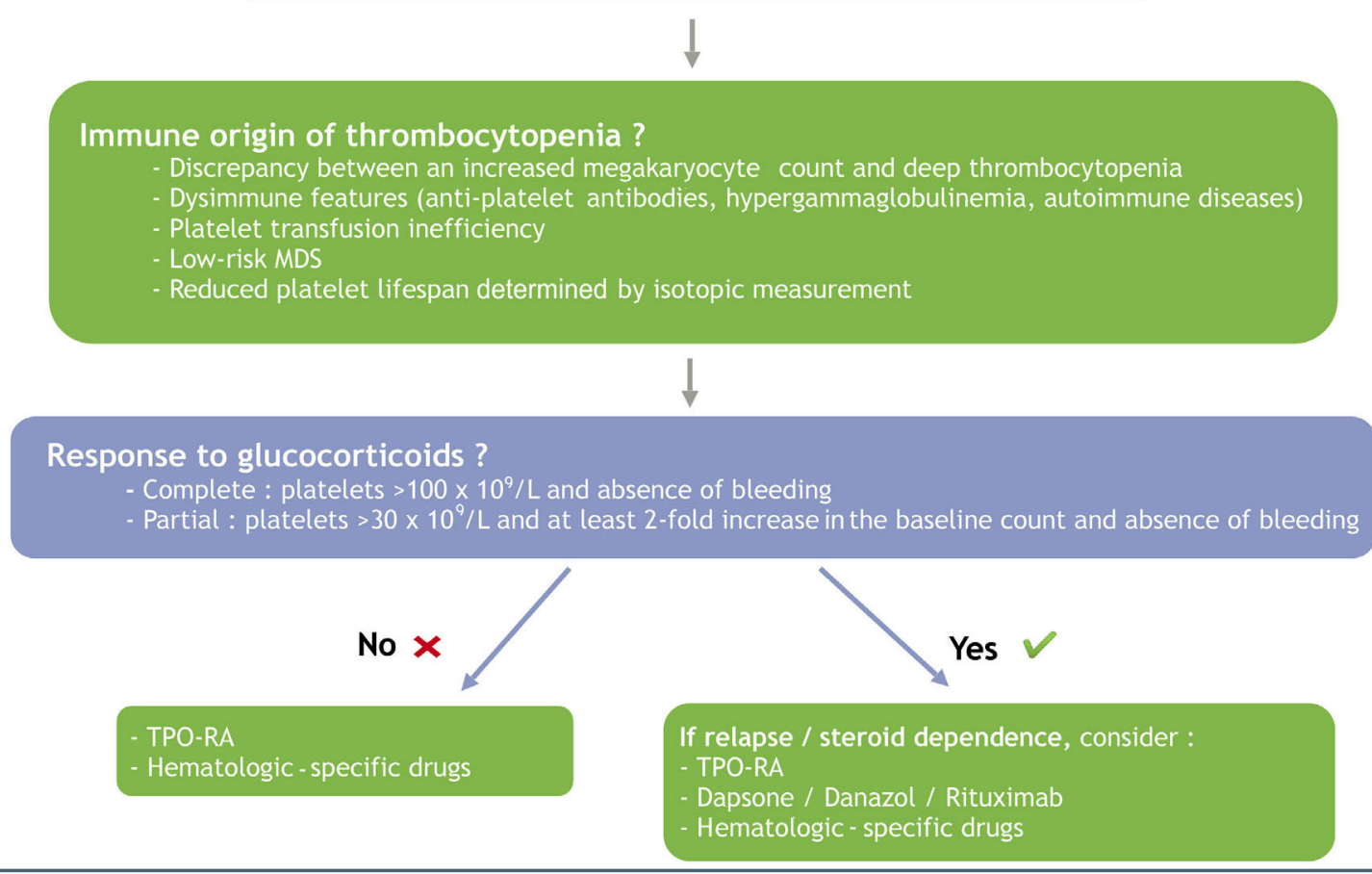

Figure 4. Proposed therapeutic strategy for suspected immune thrombocytopenia associated with myelodysplastic syndrome or chronic myelomonocytic leukemia MDS: myelodysplastic syndrome; CMML: chronic myelomonocytic leukemia; TPO-RA: thrombopoietin receptor agonist. 
MDS/CMML-associated SIAD (NCT02985190).

The prognostic significance of MDS-associated SIAD remains controversial., ${ }^{3,414-44}$ In our study, we found no difference in overall survival between MDS/CMML patients with or without ITP. However, leukemia-free survival was significantly better in MDS/CMML patients with ITP than in MDS/CMML patients without ITP.

Our study has inherent limitations considering its retrospective design, but the main limitation is the difficulty in ascertaining the immune origin of thrombocytopenia in MDS/CMML. We believe that our very strict definition of ITP in MDS/CMML patients limited the patients with a predominant central, non-immunological origin of thrombocytopenia.

In conclusion, patients with MDS/CMML-associated ITP have more severe bleeding than those with primary ITP and have a multirefractory profile, but a lower progression rate toward AML.

\section{Disclosures}

GM received research grants form CSL Behring and Novartis for the CARMEN registry in 2016 and 2017-2018. These sponsors had no role in the data collection and are not the owners of the data; they had no role in the conception, methodology, analyses and interpretation of the studies conducted in the registry and the manuscript was not submitted to the sponsors before publication. GM also received research grants from Novartis, Grifols, the Institut Servier and meeting attendance grants from Amgen and Novartis.

\section{Contributions}

$V J, G M, P F, O F$ and $A M$ designed the research and analyzed data and then wrote the paper. JH, JS, KL, NS, NV, KS, BG, $O D R, R B, J B, N B, T C, C G, O L, L L C, P T, F R P, C S, M V, F I$, $C S, M V, D G$ and JEK provided patients' data, participated in the data analysis and reread the manuscript.

\section{Appendix}

MINHEMON: French Network of Dysimmune Disorders Associated with Hemopathies; GFM: Groupe Francophone des Myélodysplasies; CERECAI: Reference Center for Autoimmune Cytopenias in Adults; CARMEN: Cytopénies Auto-immunes: Registre Midi-PyrénéEN; CARMEN investigators group: Daniel ADOUE, Laurent ALRIC, Sophie ARISTA, Laurent BALARDY, Sarah BETRIAN, Cécile BOREL, Delphine BONNET, Delphine BRECHEMIER, Miguel CARREIRO, Brice CASTEL, Thibault COMONT, Pierre COUGOUL, Alina DANU, Claire DINGREMONT, Francis GACHES, MarieHélène GASPARD, Aurélie GODEL-LABOURET, Patrick GIRAUD, Sondess HADJ-KHELIFA, Benjamin HEBRAUD, Sarah KHATIBI, Lorraine LEPLAY, Yann LEVENEUR, Sylvie OLLIER, Serge MADAULE, Clothilde MARTEL, Guillaume MARTIN-BLONDEL, Martin MICHAUD, Julia MOEGLIN, Philippe MONTANÉ de la ROQUE, Fanny NUCCIO, Maire-Léa PIEL-JULIAN, Laurent PRUDHOMME, Grégory PUGNET, Christian RECHER, Véronique REMY, Laurent SAILLER, Stéphane SIRE, Agnès SOMMET, Suzanne TAVITIAN, Willy VAILLANT.

\section{References}

1. Adès L, Itzykson R, Fenaux $P$. Myelodysplastic syndromes. Lancet. 2014;383(9936):2239-2252.

2. Grignano E, Jachiet V, Fenaux P, Ades L, Fain $\mathrm{O}$, Mekinian A. Autoimmune manifestations associated with myelodysplastic syndromes. Ann Hematol. 2018;97(11): 2015-2023

3. Seguier J, Gelsi-Boyer V, Ebbo M, et al. Autoimmune diseases in myelodysplastic syndrome favors patients survival: a case control study and literature review. Autoimmun Rev. 2019;18(1):36-42.

4. Komrokji RS, Kulasekararaj A, Al Ali NH, et al. Autoimmune diseases and myelodysplastic syndromes. Am J Hematol. 2016;91 (5):E280-283.

5. Ustwani OA, Ford LA, Sait SJN, et al. Myelodysplastic syndromes and autoimmune diseases - case series and review of literature. Leuk Res. 2013;37(8):894-899.

6. Rodeghiero F, Stasi R, Gernsheimer T, et al. Standardization of terminology, definitions and outcome criteria in immune thrombocytopenic purpura of adults and children: report from an international working group. Blood. 2009;113(11):2386-2393.

7. Hadjadj J, Michel M, Chauveheid M-P, Godeau B, Papo T, Sacre K. Immune thrombocytopenia in chronic myelomonocytic leukemia. Eur J Haematol. 2014;93(6):521526.

8. Arber DA, Orazi A, Hasserjian R, et al. The 2016 revision to the World Health Organization classification of myeloid neoplasms and acute leukemia. Blood. 2016;127(20):2391-2405.

9. Pfeilstöcker M, Tuechler H, Sanz G, et al. Time-dependent changes in mortality and transformation risk in MDS. Blood. 2016;128(7):902-910

10. Khellaf M, Michel M, Schaeffer A, Bierling P, Godeau B. Assessment of a therapeutic strategy for adults with severe autoimmune thrombocytopenic purpura based on a bleeding score rather than platelet count. Haematologica. 2005;90(6):829-832.

11. Neunert C, Noroozi N, Norman G, et al. Severe bleeding events in adults and children with primary immune thrombocytopenia: a systematic review. J Thromb Haemost. 2015;13(3):457-464.

12. Mahévas M, Gerfaud-Valentin M, Moulis $G$, et al. Characteristics, outcome, and response to therapy of multirefractory chronic immune thrombocytopenia. Blood. 2016;128(12):1625-1630.

13. Moulis G, Sailler L, Adoue D, LapeyreMestre M. Pharmacoepidemiology of immune thrombocytopenia: protocols of FAITH and CARMEN studies. Therapie. 2014;69(5):437-448.

14. Moulis G, Germain J, Comont T, et al. Newly diagnosed immune thrombocytopenia adults: clinical epidemiology, exposure to treatments, and evolution. Results of the CARMEN multicenter prospective cohort. Am J Hematol. 2017;92(6):493-500.

15. Cheson BD, Greenberg PL, Bennett JM, et al. Clinical application and proposal for modification of the International Working Group (IWG) response criteria in myelodysplasia. Blood. 2006;108(2):419425.

16. Bourgeois E, Caulier MT, Rose C, Dupriez B, Bauters F, Fenaux P. Role of splenectomy in the treatment of myelodysplastic syndromes with peripheral thrombocytopenia: a report on six cases. Leukemia. 2001;15(6):950-953.

17. Moulis G, Palmaro A, Montastruc J-L,
Godeau B, Lapeyre-Mestre M, Sailler I. Epidemiology of incident immune thrombocytopenia: a nationwide populationbased study in France. Blood. 2014;124(22):3308-3315.

18. Braun T, de Botton S, Taksin A-L, et al Characteristics and outcome of myelodysplastic syndromes (MDS) with isolated 20q deletion: a report on 62 cases. Leuk Res. 2011;35(7):863-867.

19. Barcellini W, Fattizzo B, Zaninoni A, et al. Clinical evolution of autoimmune cytopenias to idiopathic cytopenias/dysplasias of uncertain significance (ICUS/IDUS) and bone marrow failure syndromes. Am J Hematol. 2017;92(3):E26-29.

20. Glenthøj A, Ørskov A, Hansen J, Hadrup S, O'Connell C, Grønbæk K. Immune mechanisms in myelodysplastic syndrome. Int J Mol Sci. 2016;17(6):944.

21. Bao W, Bussel JB, Heck S, et al. Improved regulatory $\mathrm{T}$-cell activity in patients with chronic immune thrombocytopenia treated with thrombopoietic agents. Blood. 2010;116(22):4639-4645.

22. Bhagat TD, Zhou L, Sokol L, et al. miR-21 mediates hematopoietic suppression in MDS by activating TGF- $\beta$ signaling. Blood. 2013;121(15):2875-2881.

23. Li W, Morrone K, Kambhampati S, Will B, Steidl U, Verma A. Thrombocytopenia in MDS: epidemiology, mechanisms, clinical consequences and novel therapeutic strategies. Leukemia. 2016;30(3):536-544

24. Najean Y, Rain JD, Billotey C. The site of destruction of autologous 111In-labelled platelets and the efficiency of splenectomy in children and adults with idiopathic thrombocytopenic purpura: a study of 578 patients with 268 splenectomies. Br J Haematol. 1997;97(3):547-550.

25. Sarpatwari A, Provan D, Erqou S, Sobnack 
R, David Tai FW, Newland AC. Autologous 111 In-labelled platelet sequestration studies in patients with primary immune thrombocytopenia (ITP) prior to splenectomy: a report from the United Kingdom ITP Registry. Br J Haematol. 2010;151(5):477487.

26. Palandri F, Polverelli N, Catani L, et al. The choice of second-line therapy in steroidresistant immune thrombocytopenia: role of platelet kinetics in a single-centre longterm study. Am J Hematol. 2014;89(11): 1047-1050.

27. Mahevas M, Van Eeckhoudt S, Moulis G, et al. Autologous 111Indium-oxinate-labelled platelet sequestration study in patients with immune thrombocytopenia treated by thrombopoietin receptor-agonists. $\mathrm{Br} \mathrm{J}$ Haematol. 2019;186(3):e44-47.

28. Giagounidis A, Mufti GJ, Fenaux P, et al. Results of a randomized, double-blind study of romiplostim versus placebo in patients with low/intermediate-1-risk myelodysplastic syndrome and thrombocytopenia. Cancer. 2014;120(12):18381846.

29. Wang ES, Lyons RM, Larson RA, et al. A randomized, double-blind, placebo-controlled phase 2 study evaluating the efficacy and safety of romiplostim treatment of patients with low or intermediate-1 risk myelodysplastic syndrome receiving lenalidomide. J Hematol Oncol. 2012;5:71.

30. Dickinson M, Cherif H, Fenaux P, et al. Azacitidine with or without eltrombopag for first-line treatment of intermediate- or high-risk MDS with thrombocytopenia. Blood. 2018;132(25):2629-2638.
31. Kantariian HM, Fenaux P, Sekeres MA, et al. Long-term follow-up for up to 5 years on the risk of leukaemic progression in thrombocytopenic patients with lower-risk myelodysplastic syndromes treated with romiplostim or placebo in a randomised double-blind trial. Lancet Haematol. 2018; 5(3):e117-126.

32. Vicente A, Patel BA, Gutierrez-Rodrigues F, et al. Eltrombopag monotherapy can improve hematopoiesis in patients with low to intermediate risk-1 myelodysplastic syndrome. Haematologica. 2020;105(12): 2785-2794.

33. Oliva EN, Alati C, Santini V, et al. Long term effects of eltrombopag treatment versus placebo for low-risk myelodysplastic syndromes with thrombocytopenia (EQoLMDS): interim results of a single-blind, randomised, controlled, phase 2 superiority trial. Blood. 2019;134(Suppl_1):3000.

34. Prica A, Sholzberg M, Buckstein R. Safety and efficacy of thrombopoietin-receptor agonists in myelodysplastic syndromes: a systematic review and meta-analysis of randomized controlled trials. $\mathrm{Br} \mathrm{J}$ Haematol. 2014;167(5):626-638.

35. Dodillet H, Kreuzer K-A, Monsef I, Skoetz N. Thrombopoietin mimetics for patients with myelodysplastic syndromes. Cochrane Database Syst Rev. 2017;9(9): CD009883.

36. Erickson-Miller CL, Chadderton A, Gibbard A, et al. Thrombopoietin receptor levels in tumor cell lines and primary tumors. J Oncol. 2010;2010:135354.

37. Will B, Kawahara M, Luciano JP, et al. Effect of the nonpeptide thrombopoietin receptor agonist eltrombopag on bone marrow cells from patients with acute myeloid leukemia and myelodysplastic syndrome. Blood. 2009;114(18):3899-3908.

38. Roth M, Will B, Simkin G, et al. Eltrombopag inhibits the proliferation of leukemia cells via reduction of intracellular iron and induction of differentiation. Blood. 2012;120(2):386-394.

39. Sugita M, Kalota A, Gewirtz AM, Carroll M. Eltrombopag inhibition of acute myeloid leukemia cell survival does not depend on c-Mpl expression. Leukemia. 2013;27(5):1207-1210.

40. Liu X-G, Liu S, Feng $Q$, et al. Thrombopoietin receptor agonists shift the balance of Fcy receptors toward inhibitory receptor IIb on monocytes in ITP. Blood. 2016;128(6):852-861.

41. Mekinian A, Grignano E, Braun T, et al Systemic inflammatory and autoimmune manifestations associated with myelodysplastic syndromes and chronic myelomonocytic leukaemia: a French multicentre retrospective study. Rheumatology. 2016;55(2):291-300.

42. Dalamaga M, Petridou E, Cook FE, Trichopoulos D. Risk factors for myelodysplastic syndromes: a case-control study in Greece. Cancer Causes Control. 2002;13(7): 603-608.

43. Marisavljević D, Kraguljac N, Rolović Z Immunologic abnormalities in myelodysplastic syndromes: clinical features and characteristics of the lymphoid population. Med Oncol. 2006;23(3):385-391.

44. Giannouli S, Kanellopoulou T, Voulgarelis M. Myelodysplasia and autoimmunity. Curr Opin Rheumatol. 2012;24(1):97-102. 